Research Paper

\title{
Scutellaria Flavonoids Effectively Inhibit the Malignant Phenotypes of Non-small Cell Lung Cancer in an Id1-dependent Manner
}

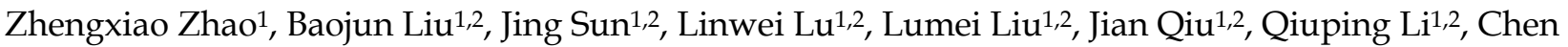 \\ Yan ${ }^{1,2}$, Shan Jiang1,2, Nabijan Mohammadtursun ${ }^{1,2}$, Wenjuan $\mathrm{Ma}^{3}$, Mihui $\mathrm{Li}^{1,2}$, Jingcheng Dong1, ${ }^{1}$, Weiyi \\ Gong1,, \\ 1. Department of Integrative Medicine, Huashan Hospital, Fudan University, Shanghai, PR China \\ 2. Institutes of Integrative Medicine, Fudan University, Shanghai, PR China \\ 3. Department of dermatology, Huashan Hospital, Fudan University, Shanghai, PR China \\ $\square$ Corresponding authors: Weiyi Gong; gongweiyi@huashan.org.cn; Tel: 13761693006; Jingcheng Dong; jcdong2004@126.com; Tel: 13601761761 \\ (c) Ivyspring International Publisher. This is an open access article distributed under the terms of the Creative Commons Attribution (CC BY-NC) license \\ (https://creativecommons.org/licenses/by-nc/4.0/). See http://ivyspring.com/terms for full terms and conditions.
}

Received: 2019.01.15; Accepted: 2019.04.23; Published: 2019.06.02

\begin{abstract}
Non-small cell lung cancer (NSCLC) is the leading cause of cancer death in the world. Inhibitor of differentiation 1 (IdI) is overexpressed in NSCLC and involved in promoting its progression and metastasis. Identifying natural compounds targeting IdI may have utility in NSCLC treatment. Here, we sought to determine whether the anti-tumor activities of Scutellaria flavonoids (SFs) were related to IdI. We reported that three SFs (baicalin, baicalein and wogonin) exhibited strong antitumor activity in NSCLC cells in vitro and in vivo. Id I played a pivotal role on blockage of migration and invasion by SFs. Abrogation of invasion and migration mediated by baicalin, baicalein and wogonin were totally abolished by ectopic overexpression of IdI. Mechanistically, baicalin, baicalein and wogonin activated Rapl-GTP binding and dephosphorylated Akt and Src by suppressing a7nAChR, consequently triggering inhibition of Idl. Then attenuation of its downstream mediators, VEGF-A, N-cadherin, vimentin, combined with augment of E-cadherin led to the blockage of proliferation, EMT and angiogenesis of NSCLC. Overall, our data shed light on heretofore-undescribed role of SFs as modulators of Idl, which may be a useful strategy in the treatment of NSCLC.
\end{abstract}

Key words: inhibitor of differentiation 1 (Id1); scutellaria flavonoids (SFs); non-small cell lung cancer (NSCLC); alpha 7 nicotinic acetylcholine receptor ( $\alpha 7 \mathrm{nAChR})$

\section{Introduction}

Lung cancer is the most commonly diagnosed cancer and leading cause of cancer death worldwide. Approximately, 1.6 million new cases and 1.4 million deaths have occurred each year [1]. Among all subtypes of lung cancer, non-small cell lung cancer (NSCLC) accounts for $80 \%$ of them. Although much progress in treating lung cancer has been made recently such as radiation therapy, chemotherapy, targeted therapy and immunotherapy, the five year survival rate remains low, only $17.7 \%$ of all patients with lung cancer are alive more than five years after diagnosis [2,3].

Inhibitor of differentiation 1(Id1) belongs to the helix-loop-helix (HLH) family of transcription factors acting as dominant negative transcriptional repressors of basic HLH (bHLH) factors [4]. Id1 can be considered as a stem cell gene, which mediates inhibition of differentiation exemplified in the maintenance of the self-renewal of embryonic stem cells and tissue progenitor cells, activation of angiogenesis, and induction of proliferation and matrix metalloproteinase-induced invasion [5]. Id1 plays a vital role in cancer biology, overexpression of Id1 has been found in a variety of human cancers including lung cancer [6] and correlated with the cancer progression and overall poor prognosis. It has been proven that Id1 is regulated through the a7 subunit of nicotine acetylcholine receptor (a7nAChR) in a panel of NSCLC cell lines and primary cells from the lung, and its down regulation abrogates NSCLC 
progression and metastasis [7,8]. In addition, Akt and Src pathways are involved in mediating Id1 to promote lung cancer cell proliferation and tumor growth $[7,9,10]$. These findings present Id1 as a promising cellular target for anti-NSCLC treatment.

A great number of herbs have been found possessing anti-tumor properties. Exploring the underlying mechanisms of them may have unexpectedly promising discoveries. Among them, Scutellaria baicalensis has been used in a wide range of cancers in China, including prostate, breast, lung, liver and ovaries cancer, etc. [11]. Baicalin, baicalein and wogonin are three major bioactive flavones derived from the root of Scutellaria baicalensis [12]. A range of chemopreventive properties of these bioactive flavones have been reported, such as apoptosis induction, autophagy triggering, cell cycle arrest, inhibition of 12-lipoxygenase and metastasis suppression [13]. Our previous study has shown that flavonoid components in Scutellaria baicalensis have potent potential against proliferation, metastasis and inflammatory microenvironment in nicotine-induced lung cancer cells [14]. And we found Scutellaria flavonoids (SFs) could inhibit nicotine-induced Id1 expression (data not shown), so that here we tried to identify mechanism of SFs linked to Id1-related pathway which might have utility in NSCLC treatment.

The results demonstrated that flavonoids in Scutellaria baicalensis, baicalin, baicalein and wogonin, effectively suppressed the growth of NSCLC in vivo and the proliferation, invasion and migration of NSCLC in vitro. Furthermore, this study presented inhibition of Id1 protein abundance via a7nAChR primarily mediated by Rap1 and in part mediated by Akt and Src, which consequently induce the suppression of proliferation, angiogenesis, and epithelial-mesenchymal transition (EMT) process of NSCLC. Our study firstly indicated that anti-cancer effects of baicalin, baicalein and wogonin were mediated through a7nAChR/Id1 pathway.

\section{Materials and Methods}

\section{Chemicals}

Baicalin and baicalein (purity > 95\%, HPLC) were purchased from Meilun Bio (Dalian, China). Wogonin (purity > 99\%, HPLC) was purchased from Desite Bio (Chengdu, China). Baicalin, baicalein and wogonin were dissolved in DMSO at $200 \mathrm{mM}, 10 \mathrm{mM}$ or $40 \mathrm{mM}$ respectively and stored at $-20^{\circ} \mathrm{C}$. 3-(4,5-dimethylthiazol-2-yl)-2,5-diphenyl-2H-tetrazoli um bromide (MTT) was from Sigma-Aldrich (Shanghai, China). Matrigel was purchased from BD Biocoat (New Jersey, USA). Cycloheximide (CHX) was from MedChem Express (New Jersey, USA). LY294002 was from Cell Signaling Technology. a-bungarotoxin(a-BT) was obtained from Abcam Company. PP2 was purchased from Enzo Biochem.

\section{Cell lines and culture}

Human NSCLC A549 cells and H1299 cells were purchased from the Chinese Academy of Sciences (Shanghai, China). A549 cells were cultured in high-glucose DMEM medium (Hyclone, Shanghai, China) and H1299 cells were grown in RPMI1640 medium (Hyclone, Shanghai, China) with 10\% fetal bovine serum (ScienCell Research Laboratories, California, USA) and $1 \%$ penicillin-streptomycin solution and incubated at $37^{\circ} \mathrm{C}$ humidified incubator containing $5 \% \mathrm{CO}_{2}$. Stable cell lines (A549-OEid1-Flag and H1299-OEid1-Flag) transduced with the Id1 gene were obtained through puromycin screening and maintained in the same complete medium supplemented additionally with $2 \mu \mathrm{g} / \mathrm{ml}$ (A549) or $1 \mu \mathrm{g} / \mathrm{ml}$ (H1299) puromycin.

\section{Cell viability and proliferation assay}

Cell viability was determined using MTT assay and cell proliferation was determined using EdU assay. Briefly, the A549 and H1299 cells seeded overnight in 96-well plates were exposed to baicalin, baicalein or wogonin at concentration of $200 \mu \mathrm{M}, 10$ $\mu \mathrm{M}$ or $40 \mu \mathrm{M}$ respectively for $24 \mathrm{~h}$. For MTT assay, MTT $(20 \mu \mathrm{L} /$ well $)$ was added to the culture at the end of treatment, and optical density was read with a wave length of $490 \mathrm{~nm}$. For the EdU assay, a Click-iT® EdU Imaging Kit (Invitrogen, Massachusetts, USA) was used. Briefly, after treatment, cells were incubated with $10 \mu \mathrm{M}$ EdU for $2 \mathrm{~h}$, then fixed with $4 \%$ paraformaldehyde and permeabilized with $0.5 \%$ Triton ${ }^{\circledR}$ X-100. EdU staining was performed according to the protocol. Cell nuclei were stained with Hoechst 33342 (Thermo Fisher Scientific Inc, Waltham, MA) at a concentration of $5 \mu \mathrm{g} / \mathrm{mL}$ for 30 minutes. More than five images of each well were taken by fluorescence microscope. Rates of EdU positive cells were analyzed.

\section{In vitro migration and invasion assay}

The in vitro migration of cells was determined using wound-healing scratch assay. Cells were grown to confluence on 6-well plates, and then each plate was scratched using a $1000 \mu \mathrm{L}$ sterile micropipette tip. The cells were washed with PBS and replaced with fresh DMEM or RPMI1640 medium, and incubated in the presence or absence of baicalin, baicalein, wogonin for $24 \mathrm{~h}$. The same wounded areas were recorded using an inverted phase-contrast microscope. In vitro invasion of cells was determined using transwell assay. Briefly, $200 \mu \mathrm{L}$ of $1 \times 10^{6}$ 
cells $/ \mathrm{mL}$ cell suspension was placed in matrigel (Corning Incorporated) coated upper chambers. $500 \mu \mathrm{L}$ DMEM or RPMI1640 medium plus 10\% FBS was added in the lower chamber as the chemo-attractant. Baicalin, baicalein or wogonin were added in the upper chamber. After 12 or 16 hours, cells on upper side of inserts were removed by using cotton, and cells on the surface of the bottom side of inserts were fixed with $4 \%$ paraformaldehyde, followed by staining with $0.5 \%$ violet. The cells in five random microscopic fields were photographed using an inverted phase-contrast microscope and counted.

\section{Lentiviral transduction in cells}

Lentiviral vectors expressing Id1 gene and a scrambled control vector, lentivirus expressing small hairpin (sh) RNAs targeting Id1 (Target sequence: GGTGAGCAAGGTGGAGATTCT) and a nontargeting short hairpin RNA control were purchased from Genechem Co (Shanghai, China). A549 cells were transfected with lentivirus for $72 \mathrm{~h}$ at multiplicity of infection (MOI):20 and H1299 cells were transfected for $48 \mathrm{~h}$ at MOI: 10. Cells were then observed under a phase-contrast fluorescence microscope to evaluate eGFP expression followed by quantitative real-time PCR and western blot analysis of Id1 expression.

\section{Quantitative real-time PCR (RT-qPCR) analysis}

After treatment or transfection, A549 and H1299 cells were isolated by trypsin/EDTA (Gibico, USA). Total RNA was extracted using Trizol reagent. cDNA synthesis was performed using RevertAid First Strand cDNA Synthesis Kit (Thermo Scientific, Waltham, USA). The Id1 mRNA expression was determined by RT-qPCR using a forward primer 5'GAGCTGAACTCGGAATCCGAA G -3' and a reverse primer 5'- GATCGTCCGCAGGAACGCATGC -3'. Then, $15 \mu \mathrm{L}$ DEPC water was added to $25 \mu \mathrm{L}$ Power SYBR Green PCR Master Mix (Life Technologies, Waltham, USA), $100 \mathrm{nM}$ primers, and $100 \mathrm{ng} / \mathrm{mL}$ cDNA. RT-PCR reaction was performed in real-time system (Applied Biosystems, Foster City, USA). The gene expression was calculated using the $\Delta \triangle \mathrm{Ct}$ method.

\section{Western blot analysis}

Treated cells or tissues were washed twice with ice-cold PBS and lysed in ice-cold assay buffer (RIPA, Beyotime, Shanghai, China) containing $100 \mu \mathrm{mol} / \mathrm{L}$ phenylmethylsulfonyl fluoride (PMSF). Protein concentrations were determined by BCA protein assay kit (Beyotime, Shanghai, China). The protein extract was mixed with SDS-PAGE sample loading buffer and heated at $100^{\circ} \mathrm{C}$ for 15 minutes. Equal amounts of protein (10-20 $\mu \mathrm{g})$ were separated by $8-12 \%$ SDS-polyacrylamide gel electrophoresis, and transferred to polyvinylidenedifluoride (PVDF) membranes. The membranes were then blocked at room temperature for one hour with $5 \%$ non-fat milk in TBST buffer and incubated with primary antibodies against Id1 (1:2000, Biocheck), $\beta$-actin (1:1000, Cell Signaling Technology), Src (1:1000, Cell Signaling Technology), p-Src (Tyr416) (1:1000, Cell Signaling Technology), AKT (pan) (1:2000, Cell Signaling Technology), p-AKT (Thr308) (1:1000, Cell Signaling Technology), p-AKT (Ser473) (1:1000, Cell Signaling Technology), a7nAChR (1:500, Abcam), N-Cadherin (1:1000, Cell Signaling Technology), E-Cadherin (1:1000, Cell Signaling Technology), VEGF-A (1:1000, Abcam) or vimentin (1:1000, Cell Signaling Technology) in 1\% Bovine Serum Albumin (BSA) overnight at $4^{\circ} \mathrm{C}$ with continuous shaking. After three washes in TBST, membranes were incubated with secondary antibodies conjugated with horseradish peroxidase for one hour and visualized by enhanced chemiluminescence using Supersignal West Femto Chemiluminescent Substrate (Pierce Biotechnology Inc., Rockford, IL, USA). Band intensities were analyzed by NIH Image software and normalized to $\beta$-actin. The western blot data was replicated three times.

\section{Immunoprecipitation}

Active Rap1 was immunoprecipitated using kit from Cell Signaling Technology (catalogue number 11877) following the manufacturer's protocol. Briefly, $1 \mathrm{mg} / \mathrm{mL}$ protein lysates were incubated with a glutathione resin and GST-RalGDS-RBD at $4^{\circ} \mathrm{C}$ for 1 $h$. The resin was then washed and incubated with reducing sample buffer $(200 \mathrm{mM}$ dithiothreitol in $2 x$ SDS sample buffer) to pull down the GTP-bound proteins. Eluents were then electrophoresed and transferred as described above and probed with rabbit anti-Rap1 (1:1000). Total Rap1 in the starting samples was also measured by Western blot.

\section{Animal experiments}

Balb/c thymic nude mice (male, 6 weeks) were obtained from B\&K Laboratory Animal Co., Ltd. (Shanghai, China). Animals were maintained under specific pathogen-free conditions with natural daylight cycles (12-hour light/12-hour dark) at $22^{\circ} \mathrm{C}$, $55 \%$ humidity and had free access to food and water. All studies were performed in accordance with the recommendations of the Guide for the Care and Use of Laboratory Animals of Fudan University and all procedures were performed under the supervision of the Animal Experimental Ethical Committee of Fudan University (Approval Number: 2015-01-HSYYDJC-01). 
To establish subcutaneous A549 xenografts, $9 \mathrm{x}$ $10^{6}$ cells suspended in $200 \mu \mathrm{L}$ PBS were inoculated in the right flank of each mouse. Tumor volumes [(length $\times$ width $\left.^{2}\right) / 2$ ] were measured by caliper twice weekly. When the tumors reached 50 to $100 \mathrm{~mm}^{3}$, mice were randomized and treatment was initiated. Baicalin $80 \mathrm{mg} / \mathrm{kg}$, baicalein $40 \mathrm{mg} / \mathrm{kg}$, wogonin 80 $\mathrm{mg} / \mathrm{kg}$ (dissolved in 0.5\% CMC-Na) or CMC-Na was administered intragastrically daily for 28 days $(n=8)$. At the end of the experiment, mice were sacrificed and tumors were harvested and weighted.

\section{Immunohistochemistry}

For immunohistochemical (IHC) analyses, paraffin-embedded tissue of mouse tumors were sectioned $(3 \mu \mathrm{m})$ using a rotary microtome (Leica, London, UK) and collected on slides. Then, the deparaffinized tissue was heated in citrate buffer to perform the antigen retrieval. Afterwards, the sections were further blocked by treatment with $5 \%$ goat serum for $1 \mathrm{~h}$ at room temperature and then incubated with the primary antibody using rabbit monoclonal IgGs specific Id1 antibody (1:100, BioCheck), VEGF-A (1:200, Abcam), CD31(1:100,Servicebio) or vimentin (1:100, Cell Signaling Technology) at $4^{\circ} \mathrm{C}$ overnight, followed by biotin labeled secondary antibody, developed with 3,3'-diaminobenzidine. For a negative control, a nonspecific antibody was used instead of the primary antigen. Staining was visualized and quantified by light microscope. Analysis of staining was performed with Image-Pro Plus software (Media Cybernetics Inc., Rockville, MD, USA).

\section{Statistical analysis}

Experiments were repeated three times. The data was presented as the mean \pm standard error (SE). The one-way ANOVA with post hoc comparison by the Dunnett's test was used to analyze the differences of multiple groups. Comparisons between two groups were analyzed by $t$ test. P-values $<0.05$ were considered statistically significant. GraphPad Prism 6.02 software (GraphPad, San Diego, CA) was used for statistical analysis.

\section{Results}

\section{SFs Inhibited the Growth of NSCLC in Vitro and in Vivo}

At first, we determined the effects of three main components of Scutellaria, baicalin, baicalein and wogonin, on growth of human NSCLC cells. A549 and H1299 cells were exposed to baicalin, baicalein or wogonin in concentration of $200 \mu \mathrm{M}, 10 \mu \mathrm{M}$ or $40 \mu \mathrm{M}$ respectively for $24 \mathrm{~h}$. Cell viabilities were determined by MTT assay. As shown in Figure 1A, baicalin, baicalein and wogonin effectively counteracted the cellular viability on both cell lines $(p<0.01)$. The inhibitory effects of baicalin, baicalein and wogonin were further validated in H1299 cells by Edu assay, and similar inhibitory effects of baicalin and baicalein on A549 cells were also observed (Figure 1B). To verify the effects of the main constitutes of Scutellaria to NSCLC in vivo, we established subcutaneous A549 xenografts in Balb/c nude mice. As shown in Figure $1 \mathrm{C}$, there was a significant difference in tumor weight between flavones-treated and vehicle-treated groups $(p<0.05)$. Tumor-bearing mice treated with baicalin, baicalein or wogonin respectively for 28 days showed reduction in tumor volume, while baicalein exerted the best inhibitory effect $(p<0.05)$. In addition, no significant difference was observed in the mice body weight between the control group and treatment groups.

\section{SFs Suppressed the Invasion and Migration of NSCLC}

Dissemination is one of main features of NSCLC which indicates poor prognosis of patients [15]. We thus investigated the role of baicalin, baicalein and wogonin on the invasion and migration of A549 and H1299 cells. Transwell chamber assay was conducted to evaluate the effects of these phytochemicals on invasion. As shown in Figure 2A, after $12 \mathrm{~h}$ incubation, baicalin, baicalein and wogonin significantly suppressed the invasive capacities both in A549 and H1299 cells ( $p<0.01)$. In parallel, wound healing assay was used to determine the effects of these components on migration. The results showed that the wound-healing abilities were dramatically inhibited after treating A549 cells with the indicated concentrations of three flavones respectively. Similarly, baicalein or wogonin treatment also significantly reduced the migratory capacity of H1299 cells (Figure 2B).

\section{SFs Repressed Expression of Id I}

Given that Id1 is involved in promoting lung cancer cell proliferation and metastasis, we explored if the bioactive constituents of Scutellaria inhibited Id1 expression [6]. The results of western blotting analyses showed that baicalin, baicalein and wogonin inhibited Id1 protein expression in a dose-dependent manner (Figure 3A). As shown in Figure 3B, incubation with these phytochemicals significantly decreased the expression of Id1 mRNA in H1299 cells, while as to A549 cells, pretreatment with them reduced the expression level of Id1 mRNA, but the differences were not significant. Therefore, we determined if baicalin, baicalein or wogonin affected the degradation of Id1 protein. Cycloheximide (CHX, $100 \mu \mathrm{g} / \mathrm{mL}$ ), a classical inhibitor of de novo protein 
synthesis, was added to A549 or H1299 cells. After A549 cells or H1299 cells were incubated in presence or absence of baicalin, baicalein or wogonin for indicated hours, Id1 protein expressions were determined. The data showed that the half-life of Id1 in A549 and H1299 cells was not accelerated by co-treatment of baicalin, baicalein or wogonin when compared to cells exposed to CHX alone (Figure 3C).

\section{Id I Played a Pivotal Role on Blockage of Migration and Invasion by SFs}

To better understand the involvement of Id1 in SFs-mediated anti-metastatic effect, A549 and H1299 cells were transduced with lentiviral shRNA or lentiviral constructs encoding human Id1 gene. Initially, western blot and RT-qPCR were performed to confirm the efficiency of transfection (Figure 4A and Figure 4B). We observed that shRNA 739 efficiently decreased the expression of Id1. Besides, about two folds of ectopic expression of Id1 was detected in cells transduced with lentivirus constructs encoding human Id1. Next, MTT assay showed that knockdown of Id1 decreased the cell viability and ectopic expression rescued this deterioration (Figure 4C). We also found that knockdown of Id1 reduced the invasion of A549 and H1299 cells and significantly decreased the distance of migration $(p<0.01)$. In contrast, overexpression of Id1 significantly boosted the invasion and migration capacity of A549 and H1299 cells $(p<0.01)$ (Figure 4D). To further verify whether Id1 is involved in the suppression of lung cancer progression by flavones of Scutellaria, Id1 was overexpressed and then A549 and H1299 cells were incubated with or without of baicalin, baicalein or

A
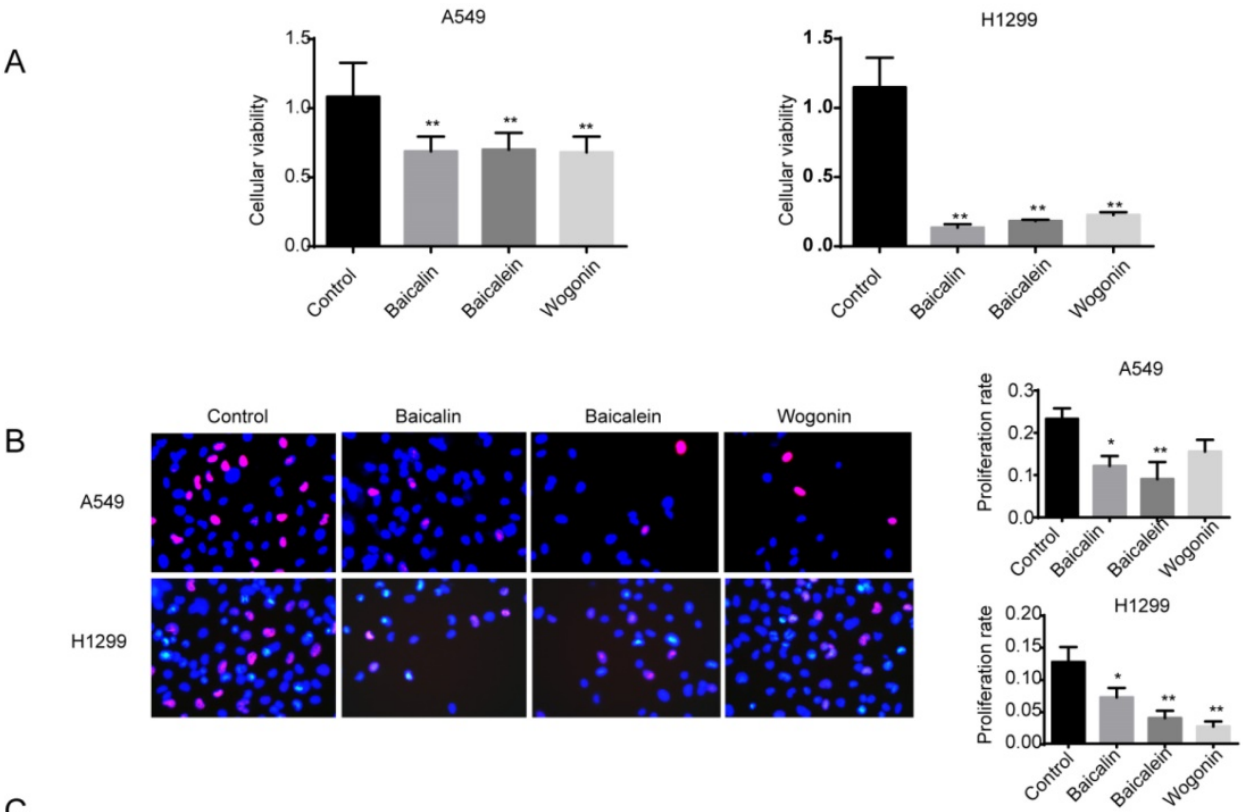

C
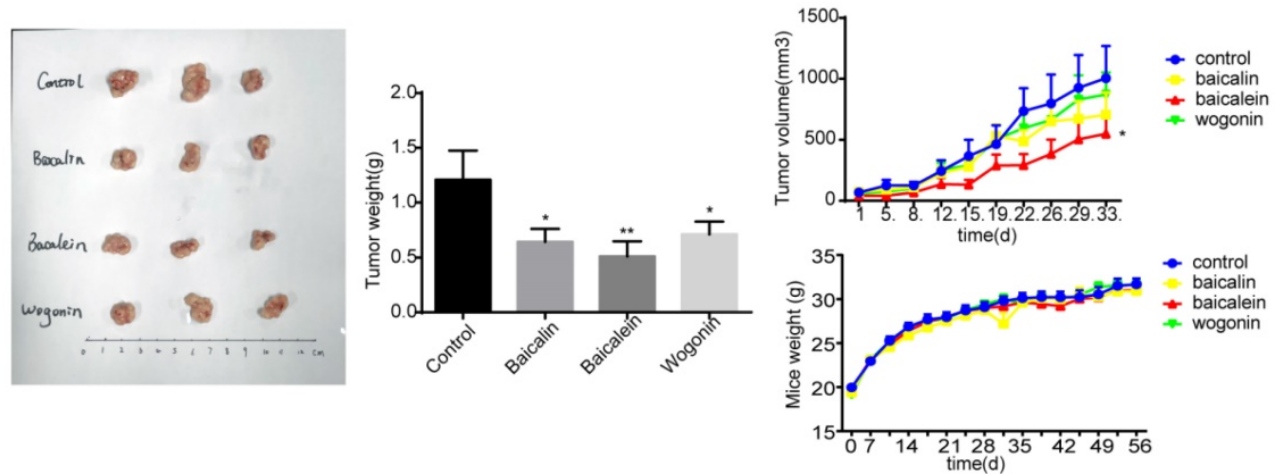

Figure 1. Baicalin, baicalein and wogonin inhibited the growth of NSCLC in vitro and in vivo. (A) A549 and H1299 were exposed to baicalin, baicalein or wogonin in concentration of $200 \mu \mathrm{M}, 10 \mu \mathrm{M}$ or $40 \mu \mathrm{M}$ respectively for $24 \mathrm{~h}$, and cell viability was determined by MTT assay. (B) A549 and H1299 were exposed to baicalin, baicalein or wogonin in concentration of $200 \mu \mathrm{M}, 10 \mu \mathrm{M}$ or $40 \mu \mathrm{M}$ respectively for $24 \mathrm{~h}$, cells were incubated with $10 \mu \mathrm{M}$ EdU for $2 \mathrm{~h}$ and EdU assay was performed. Five images of each well and three duplicated wells were taken by fluorescence microscope and EdU positive cells were counted. (C) Balb/c nude mice bearing palpable A549 xenografted tumors were intragastrically administered with control (CMC-Na), baicalin $(80 \mathrm{mg} / \mathrm{kg})$, baicalein $(40 \mathrm{mg} / \mathrm{kg})$ or wogonin $(80 \mathrm{mg} / \mathrm{kg})$ daily. Representative tumors and tumor weights dissected on day 28 after treatment were shown (left). The tumor volumes and mice weight measured twice a week versus time was plotted (right) ( $\mathrm{n}=8)$. Each bar represented the mean \pm SEM. Significant differences were shown $\left({ }^{*} p<0.05\right.$ and $*^{* *} p<0.01$, compared with the control group). 
wogonin. In A549 cells, after $24 \mathrm{~h}$ treatment, abrogation of invasion and migration mediated by baicalin, baicalein or wogonin were totally reversed by ectopic overexpression of Id1 (Figure 4E). In H1299 cells, invasion chamber assay showed that the inhibition of invasion mediated by baicalin or baicalein were reversed by overexpression of Id 1 after $16 \mathrm{~h}$ treatment (Figure S1A). And woundhealing assay showed that after $24 \mathrm{~h}$ treatment, inhibition of migration mediated by baicalin, baicalein or wogonin were attenuated when Id1 was overexpressed (Figure S1B).

\section{Angiogenesis and EMT were Further Attenuated by SFs}

To investigate the downstream signaling pathway of Id1, several important molecules including VEGF-A, N-cadherin, E-cadherin and vimentin in A549 and H1299 cells were determined. After transiently transfected with Id1 shRNA construct, the expression of VEGF-A, N-cadherin and vimentin was decreased remarkably and the expression of E-cadherin was highly up-regulated, while opposite results were observed in
Id1-overexpressed cells. $(p<0.01)$ (Figure 5A). To further evaluate the effects of baicalin, baicalein and wogonin on these downstream molecules, A549 and H1299 cells were incubated with these phytochemicals respectively for $24 \mathrm{~h}$. Western blot analyses showed that all components significantly inhibited the expression of Id1, VEGF-A, N-cadherin, vimentin in A549 cells $(p<0.01)$. Moreover, more E-cadherin protein expression was observed in baicalein- or wogonin- regulated A549 cells $(p<0.01)$. In H1299 cells, there were significant decreases in the amounts of Id1, N-cadherin, vimentin proteins and increases in the level of E-cadherin in three flavones treated cells, compared with those in control cells $(p$ $<0.01$ ). As for VEGF-A, baicalein and wogonin substantially blocked its expression $(p<0.01)$, but similar inhibitory effect was not observed in baicalintreated cells (Figure 5B). Next, to confirm these effects of SFs in vivo, we evaluated these molecules in A549 subcutaneous tumor model. As shown in Figure 5C, baicalin, baicalein and wogonin dramatically suppressed expressions of Id1, VEGF-A, N-cadherin and vimentin, facilitated E-cadherin protein expression in tumor tissue $(p<0.01)$. Consistently,

A
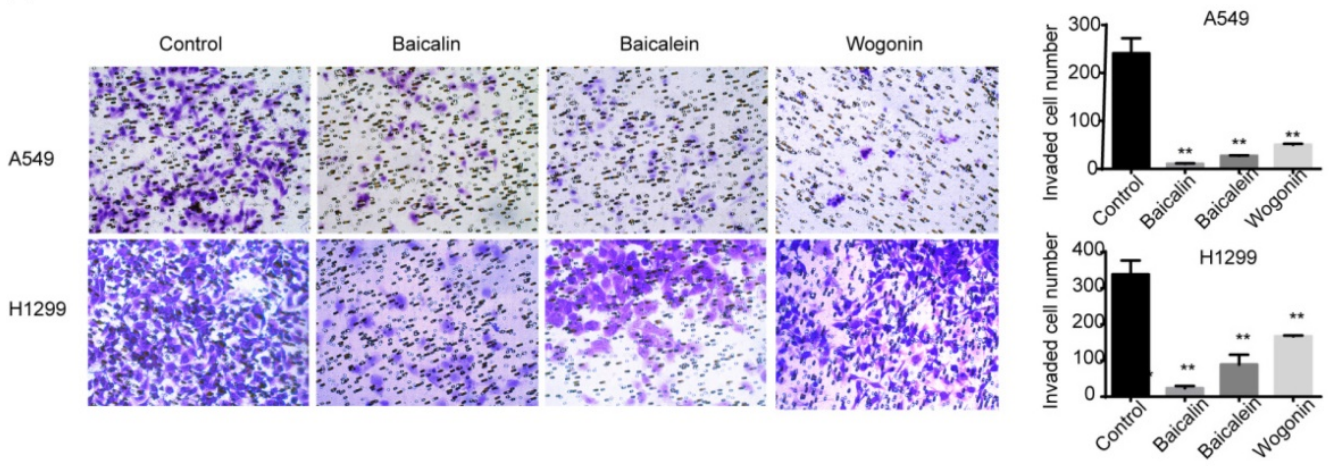

B

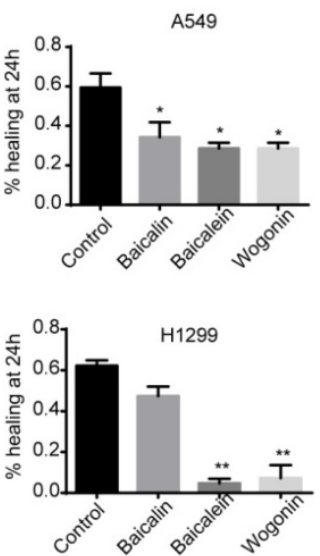

Figure 2. Baicalin, baicalein and wogonin suppressed the invasion and migration of NSCLC. (A) A549 and H1299 cells were treated in the presence or absence of baicalin, baicalein or wogonin for $12 \mathrm{~h}$, and subjected to matrigel invasion chamber assay. The invaded cells were calculated in three random fields per experiment. (B) A549 and H1299 were cultured in 6-well plates and scratches were created using sterile pipette tips. After $24 \mathrm{~h}$ incubation with or without baicalin, baicalein or wogonin, records were taken using microscope. Three duplicated experiments were performed. Representative scratches for A549 and $\mathrm{H} 1299$ were shown; the breadth of scratches was measured and bar graphs were the quantitative analysis of percentage of wound closure relative to the breadth of beginning from three random microscopic fields at the indicated time points. Data represented mean \pm SEM. $*, p<0.05 ; * *, p<0.01$. 


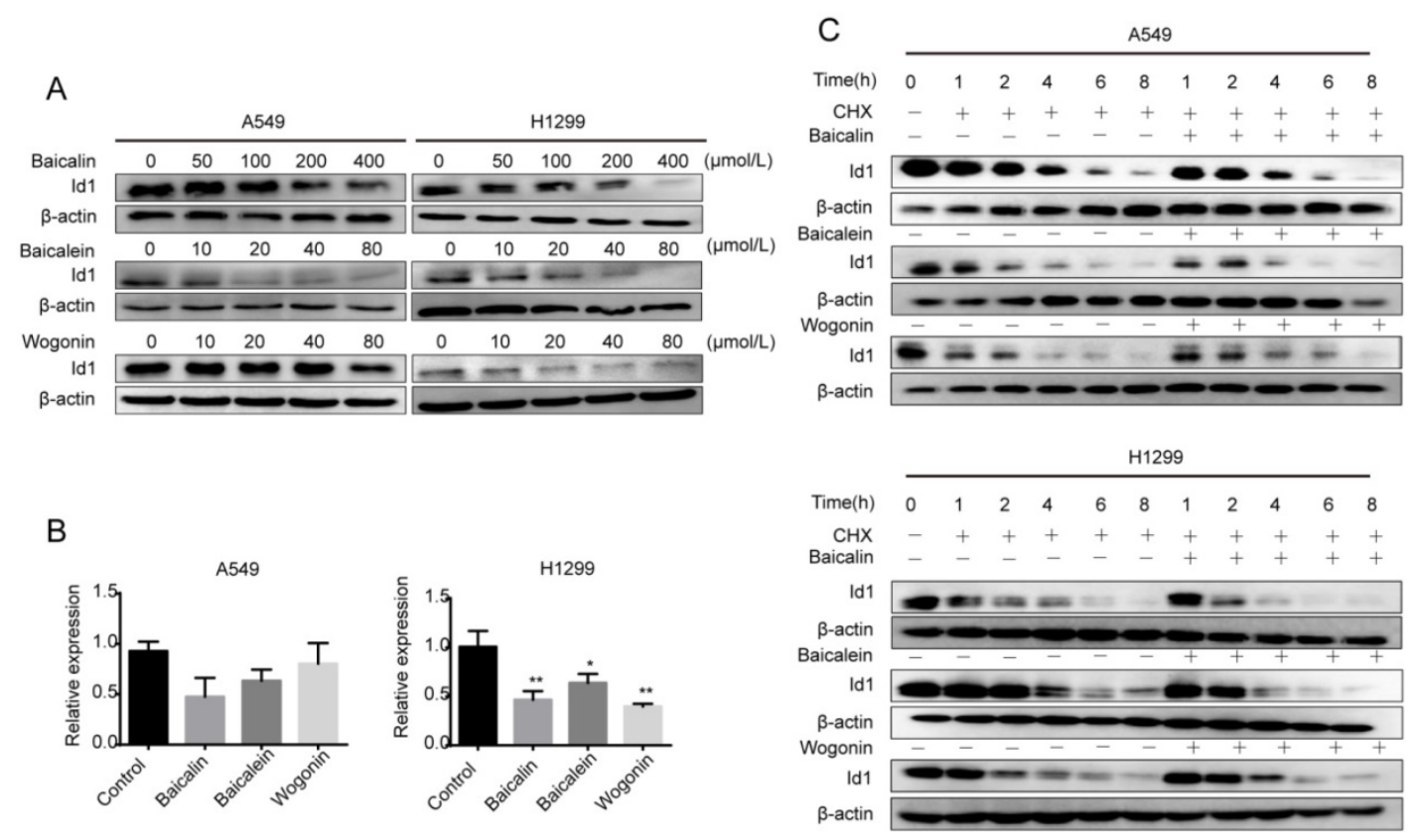

Figure 3. Baicalin, baicalein and wogonin repressed expression of Idl. (A) A549 or H1299 cells were exposed to increasing concentrations of baicalin, baicalein or wogonin for $24 \mathrm{~h}$. Idl expression level was analyzed by western blot. (B) A549 or H1299 cells were exposed to baicalin, baicalein or wogonin at concentration of $200 \mu \mathrm{M}, 10 \mu \mathrm{M}$ or $40 \mu \mathrm{M}$ respectively for $24 \mathrm{~h}$. RT-PCR analysis was performed to determine the expression of Idl mRNA. (C) Time-course studies of Idl degradation were performed by adding cycloheximide $(\mathrm{CHX}, 100 \mu \mathrm{g} / \mathrm{mL})$ to $\mathrm{A} 549$ or $\mathrm{H} 1299$ cells in presence or absence of baicalin, baicalein or wogonin $(200 \mu \mathrm{M}, 10 \mu \mathrm{M}$ or $40 \mu \mathrm{M})$ for $0,1,2,4,6,8 \mathrm{~h}$, followed by western blot assay. Data represented mean \pm SEM. *, $p<0.05 ; * *, p<0.01$.

IHC showed that three components effectively suppressed Id1 expression. Wogonin inhibited expression of VEGF-A $(p<0.01)$ and staining of CD31 showed that microvessels density (MVD) of tumors was dramatically inhibited by baicalin, baicalein and wogonin $(p<0.01)$. As to vimentin, all three phytochemicals repressed its expression but the inhibition of baicalin was not striking (Figure 5D). Additionally, to confirm whether other components were involved in the inhibitory effects of SFs on these downstream, Stable cell lines (A549-OEid1-Flag and H1299-OEid1-Flag) transduced with the Id1 gene were treated with baicalin, baicalein or wogonin. Actually, the effects of SFs to these downstream molecules were abrogated by ectopic expression of Id1 completely (Figure 5E).

\section{Id I Inhibition of SFs was Through Restraining of Phosphorylation of Akt/Src and Suppression of $\alpha 7 n A C h R$}

To elucidate how SFs inhibited Id1 expression, we investigated the role of a7nAChR, Akt and Src in the inhibitory effect of SFs, as studies show that a7nAChR, Akt and Src are critical in NSCLC [16-18]. We first examined the relationship between a7nAChR, Akt or Src and Id1. When A549 cells and H1299 cells exposed to $5 \mu \mathrm{M}$ or $10 \mu \mathrm{M}$ a7nAChR antagonist, a-bungarotoxin (a-BT), for $24 \mathrm{~h}$, Id1 expression was significantly impaired. In addition, after PP2 (Src kinase inhibitor) treatment, Id1 protein levels were down-regulated in dose- dependent manner in $\mathrm{H} 1299$ cells and attenuated by $50 \mu \mathrm{M}$ for 48 $\mathrm{h}$ in A549 cells. When cells were treated with PI3K/Akt inhibitor, LY294002, Id1 expression was also inhibited in a dose-dependent way (Figure 6A). These results indicated that a7nAChR, Akt and Src were upstream of Id1. As a study reported Id1 was upstream of Akt which was contradictory to our study [9]. We then examined the effects of knockdown and overexpression of Id1 on a7nAChR, Akt and Src. Results showed that when Id1 was overexpressed or knocked down in A549 cells, effects on expressions of a7nAChR, Akt, Src or phosphorylation of Akt, Src were not observed (Figure S2A). In H1299 cells, the expressions of a7nAChR, T-akt, Src and phosphorylation of Akt and Src were significantly suppressed when Id1 was overexpressed. Meanwhile, when Id1 was knocked down, the phosphorylation of Akt was significantly upregulated, however, T-akt was downregulated and expressions of a7nAChR, Src, p-Src(Tyr416) were not affected (Figure S2B). Furthermore, we examined the effects of baicalin, baicalein and wogonin on these upstream molecules. Western blot analysis showed that baicalin, baicalein and wogonin dramatically reduced the expression of a7nAChR in A549 and H1299 cells ( $p<0.01)$. Similarly, the inhibitory effects of phosphorylation of Src (p-Src(Tyr416)) by these three components on both cell lines were also observed. Reduction of Src was significant when A549 cells treated with baicalin and H1299 cells treated with baicalein or wogonin. Moreover, baicalin, baicalein and wogonin were 
responsible for decreasing the expressions of p-Akt (Thr308) and p-Akt (Ser473) as well $(p<0.01)$ (Figure $6 \mathrm{~B})$.

\section{a7nAChR Regulated Rapl Activation and Src/Akt Dephosphorylation}

As KEGG pathway analysis suggested that Rap1 regulates Src, Akt and Id1 expression (data not shown), we aimed to identify the effects of baicalin, baicalein and wogonin on Rap1. Rap1 is a switch molecule belonging to Ras family of GTPase and famous for its ability to normalize a malignant phenotype of KRAS transformed fibroblasts [19]. Rap1 activation has been implicated in regulating cell adhesion, polarity, and various integrin-mediated biological progresses [20,21]. Our results showed that baicalin, baicalein and wogonin effectively promoted the GTP-loaded Rap1 ( $p$ <.01) (Figure 7A).

Mutation of Ras gene is predominant in tumorigenesis of lung cancer, and it has been reported that Ras protein is mainly regulated by $\mathrm{nAChR}$ [22]. Considering that Rap protein shares the great sequence similarity with Ras proteins [23], we presumed that Rap1 was regulated by a7nAChR. Pre-treatment with $10 \mu \mathrm{M}$ a-BT for $24 \mathrm{~h}$ caused an elevation of Rap1-GTP and reduction of both p-Akt (Ser473) and p-Src (Tyr416) $(\mathrm{p}<0.01)$ (Figure 7B). To further investigate the relationship among Rap1, Src, Akt and Id1, we activated Rap1 by 8-pCPT-2'-O-Me-cAMP (8-pCPT) and then determined the expression of Src, Akt and Id1. Unexpectedly, different from KEGG pathway analysis, we found that Rap1 activation exhibited a decreased ability to Id1 expression, but had no effect on Src and Akt phosphorylation (Figure 7C).

\section{Discussion}

Our current results demonstrated that bioactive constituents (baicalin, baicalein and wogonin) in Scutellaria effectively inhibited the growth, migration and invasion of NSCLC by mediating Id1-related signaling pathway. Importantly, Rap1 activation and dephosphorylation of $\mathrm{Src}$ and Akt regulated by a7nAChR were responsible for Id1 suppression by these phytochemicals and its down-regulation largely affected downstream mediators (VEGF-A, $\mathrm{N}$-cadherin, E-cadherin and vimentin). In view of these observations, our findings provide novel insights into the anti-tumor molecular mechanisms of SFs and propose Id 1 and its mediators as therapeutic targets for anti-tumor strategies.

Scutellaria baicalensis exerts multiple biological functions, including anti-inflammatory, anti-oxidant and anti-cancer properties [24-26]. Among more than 40 flavonoids derived from Scutellaria baicalensis, studies focus more intensively on components including baicalein, baicalin, wogonin [26]. Previous studies have shown that flavonoids in Scutellaria baicalensis inhibit lung cancer via SIRT1/AMPK, Notch or PTEN/PI3K/AKT pathway, etc. [27-29]. We tried to examine the effects of baicalin, baicalein and wogonin on Id1-mediated signaling pathways in lung cancer. We found that SFs effectively inhibited proliferation and mobility of NSCLC cells (Figure 1 and Figure 2), which related to down-regulation of Id1 expression but not to its degradation (Figure 3). We noticed that mRNA expression of Id1 markedly affected by flavones was consistent with the corresponding protein expression in $\mathrm{H} 1299$ cells, but not in A549 cells, implying potentially separate isoform-specific mechanisms for the regulation of protein abundance [30].

Id1 is a member of helix-loop-helix (HLH) transcription factors that displays no DNA-binding motif, thus inhibits DNA binding of other HLH proteins and cell differentiation [6]. It has been implicated in several cellular processes, including growth, differentiation and senescence and is overexpressed in multiple cancers [4,31-33]. In agreement with previous studies, our study also showed the crucial role of Id1 in NSCLC, as studies conducted on Id1-knockdown cells led to decreased cell proliferation and cancer invasion, while overexpression of Id1 significantly reversed these inhibitory effects. Moreover, we provided evidence that overexpression of Id1 totally abolished SFs-induced inhibitory effect on invasion and migration (Figure 4), suggesting that inhibition of Id1 may contribute to the anti-metastatic effects of SFs.

It is well known that EMT and neoangiogenesis are two critical physiological features which facilitate metastasis of tumor. It has been reported that Id1 enables lung cancer liver colonization through activation of EMT program in tumor cells and establishment of the pre-metastatic niche [34]. Additionally, studies show angiogenesis is regulated by Id1 pathway in women with endometriosis and gastric cancer $[35,36]$, but Id1 is shown up-regulated by VEGF-A, one of the most critical factors that stimulates angiogenesis, in atherosclerotic plaque rupture [37]. In our study, we found that baicalin, baicalein and wogonin effectively inhibited the expression of $\mathrm{N}$-cadherin, vimentin and up-regulated E-cadherin, they also decreased MVD in vivo and reduced the expression of VEGF-A in vitro and in vivo. In addition, overexpression of Id1 eradicated the effects of baicalin, baicalein and wogonin on these molecules (Figure 5), indicating that Id1 was involved in the inhibition of EMT procedure and neoangiogenesis by SFs. 
A

Id1

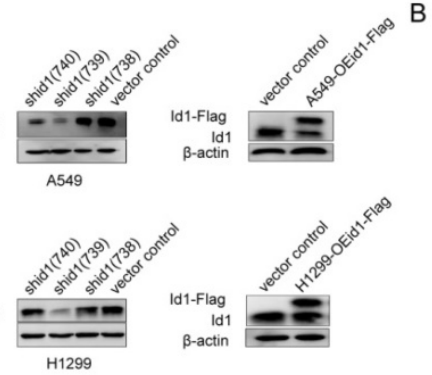

C

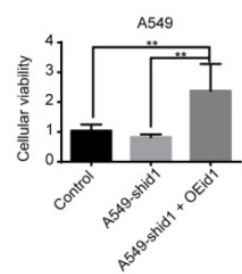

D
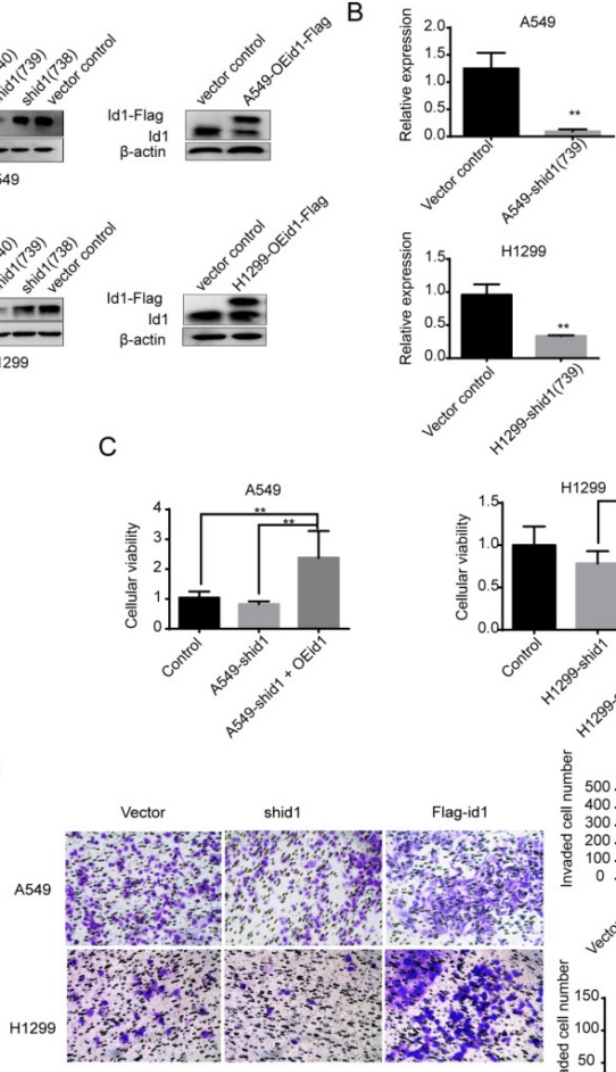

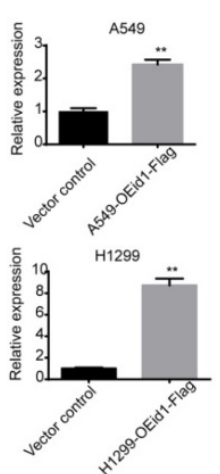

(1.5)

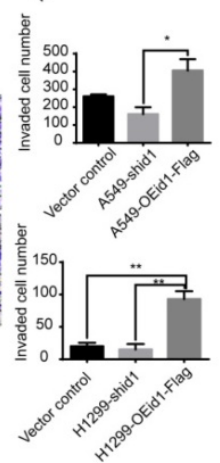

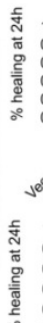

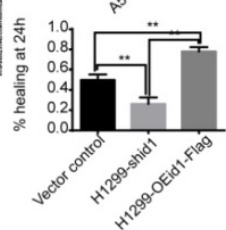

A549
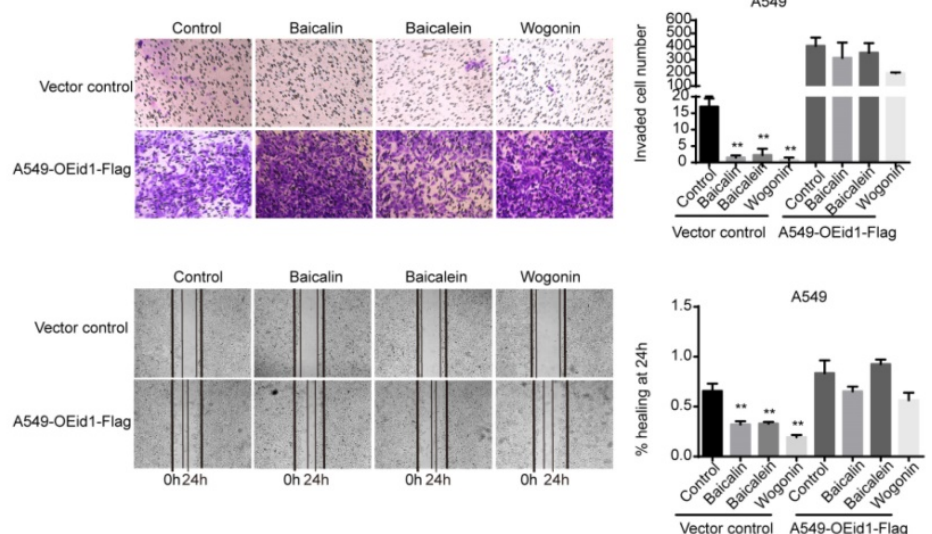

Figure 4. Id I played a pivotal role on blockage of migration and invasion by SFs. (A) A549 and H1299 cells were transfected with either lentivirus Id I-shRNA constructs or Id I cDNA, and confirmed by western blot and (B) RT-qPCR analysis. (C) A549 and H1299 cells were transfected with lentivirus Id1-shRNA constructs and then transfected with or without constructs expressing IdI; MTT assay was performed to determine the cell viability. (D) A549 and H1299 cells were transfected with either lentivirus IdI-shRNA constructs or IdI cDNA, and then subjected to matrigel invasion chamber assays for $12 \mathrm{~h}$ or to wound healing assay for $24 \mathrm{~h}$. (E) A549 cells were transfected with control vector or construct expressing Id l, and treated with or without baicalin, baicalein or wogonin for $24 \mathrm{~h}$. Invasion chamber assay and wound healing assay were conducted. The relative quantitative determinations of migrated area or invaded cells were calculated with five fields counted per experiment. Data represented mean \pm SEM. $*, p<0.05 ; * *, p<0.01$. 


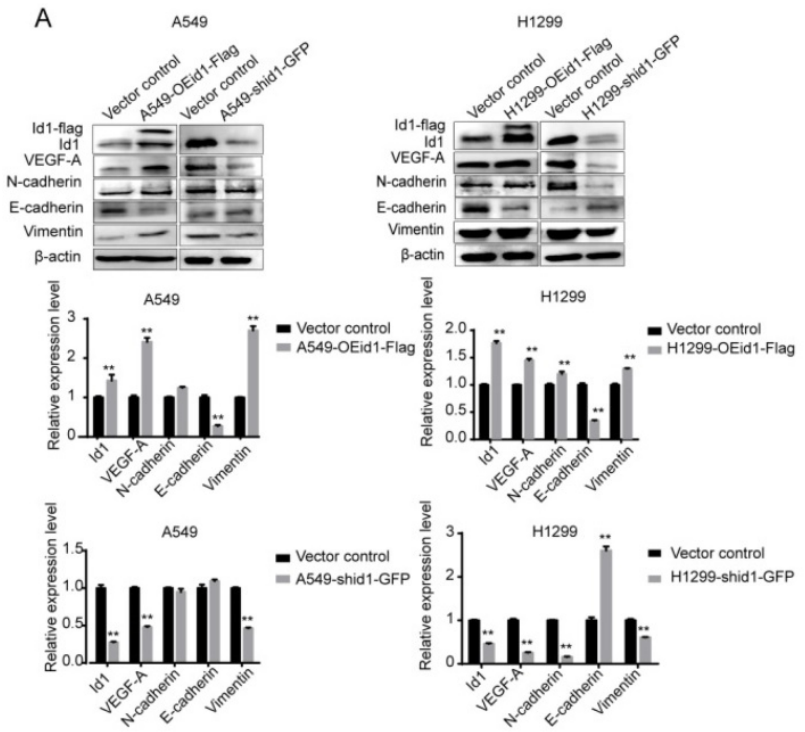

B
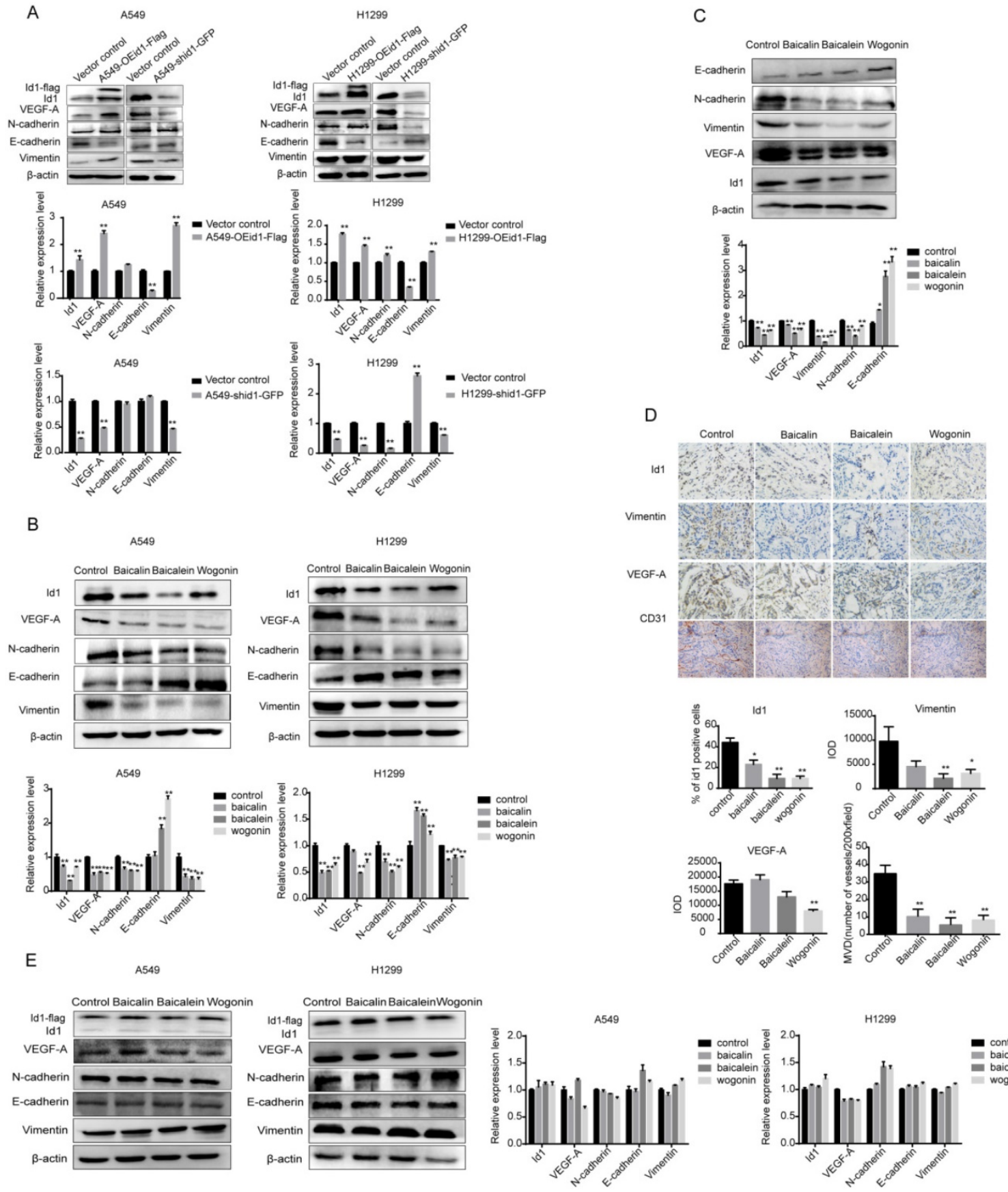

Figure 5. Angiogenesis and EMT procedures were suppressed by baicalin, baicalein and wogonin. (A) A549 and H1299 cells were transfected with either Id I cDNA or lentiviral Id I-shRNA constructs to overexpress or knockdown Idl gene and expressions of VEGF-A, N-cadherin, E-cadherin and vimentin were determined by western blot analysis. (B) A549 and H1299 cells were treated with baicalin, baicalein or wogonin at concentration of $200 \mu \mathrm{M}, 10 \mu \mathrm{M}$ or $40 \mu \mathrm{M}$ respectively for $24 \mathrm{~h}$, and expression levels of Id 1 , VEGF-A, $\mathrm{N}$-cadherin, E-cadherin and vimentin were determined by western blot analysis. (C) After treated by baicalin, baicalein or wogonin for 28 days, subcutaneous xenografts were sacrificed and tumors were lysed and electrophoresed. Expression levels of IdI, VEGF-A, N-cadherin, E-cadherin and vimentin were determined ( $n=5)$. (D) Tumor tissues were fixed and IHC was conducted to examine the levels of IdI, VEGF-A, CD3I and vimentin (n=3). Bar graphs were quantitative analyses of positive cells or integral optical density (IOD). Six slides of each group were evaluated at 100x magnification to identify the "hot-spot" regions of CD31 staining. Five most vascularized areas were selected and counted at 200x magnification as numbers of microvessels (MVD). (E) A549-OEidl-Flag and H1299-OEid1-Flag cells were treated with baicalin, baicalein or wogonin for 24 h. Data represented mean \pm SEM. *, $p<0.05 ; * *, p<0.01$.

However, the mechanism by which SFs regulate Id1 expression is not yet clear. nAChR are expressed on normal bronchial epithelial and NSCLC cells and involved in cell growth regulation [38], while inhibition of non-neuronal a7nAChR reduces tumorigenicity in A549 NSCLC xenografts[16]. Strong positive correlations between Id1 and $\mathrm{a} 7 \mathrm{nAChR}$ are observed in human tumor samples, and studies in vitro prove that Id1 induction is mediated through a7nAChR $[7,8,39]$. Indeed, Src and Akt pathways are also found to be effective in regulating Id1 function. Src is a famous tyrosine kinase involving in multiple aspects of tumorigenesis including proliferation, migration and angiogenesis [17]. Microarray analyses 
show that Id family genes were among the most highly down-regulated by Src inhibitor AZD0530 [40]. Likewise, Akt activation is a poor prognostic factor for all stages of NSCLC [18]. Our study showed that inhibitors of a7nAChR, Src and Akt (a-BT, PP2 and LY294002) dose-dependently suppressed the expression of Id1 (Figure 6) which was consistent with above studies. In addition, we found that negative feedback might exist in H1299 cells as results showed
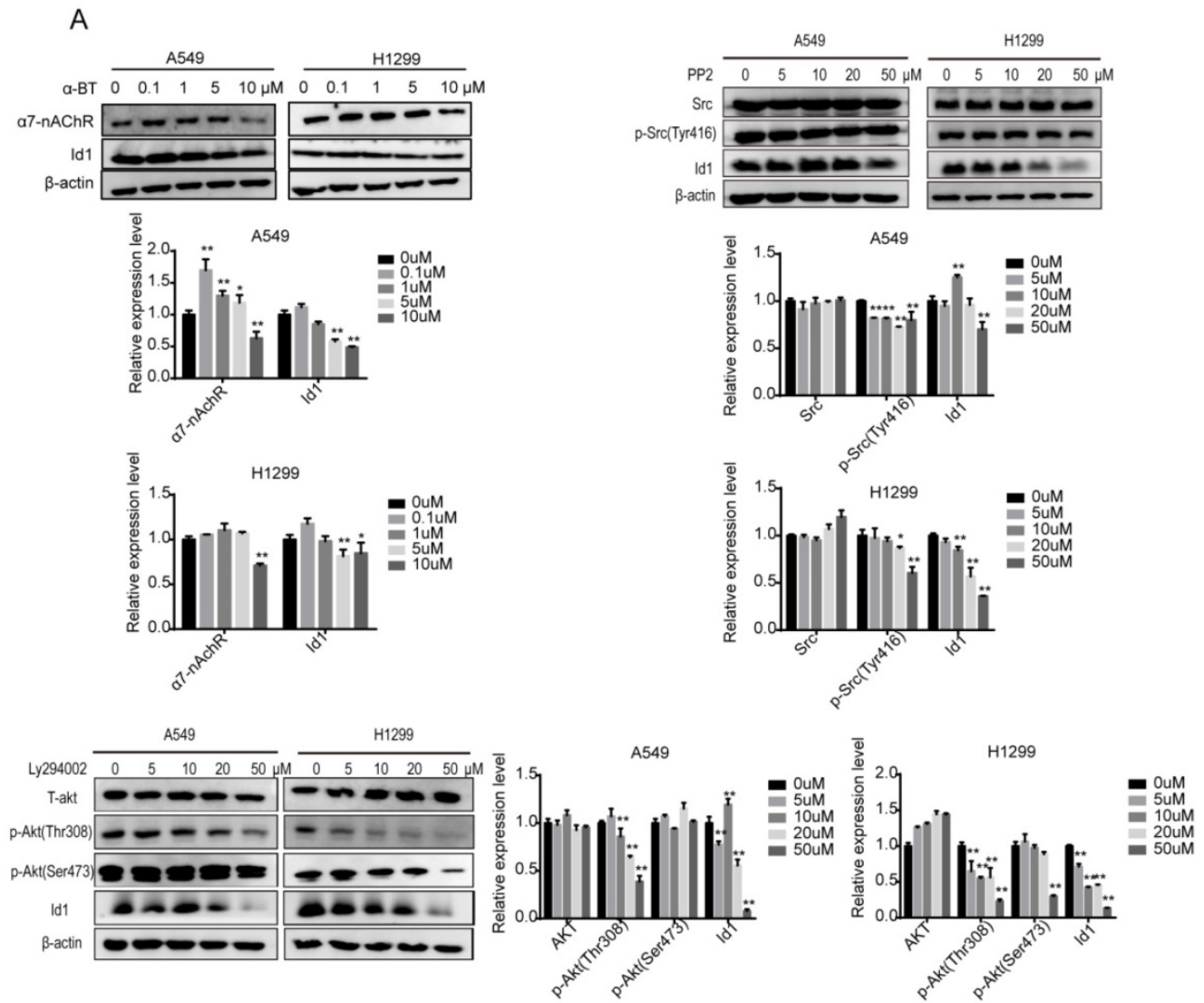

B

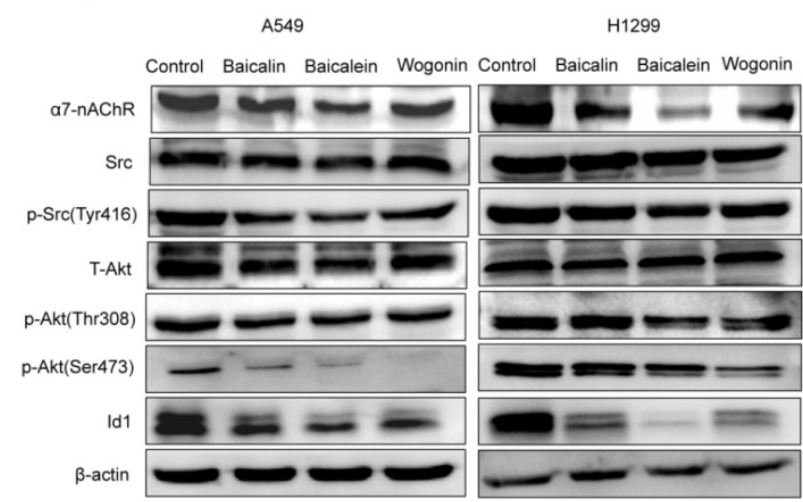

overexpression of Id1 significantly inhibited the expression of a7nAChR, Src and Akt and phosphorylation of latter two (Figure S2). Moreover, baicalin, baicalein and wogonin significantly suppressed the expression of $\mathrm{a} 7 \mathrm{nAChR}$ and phosphorylation of Src and Akt, which suggested that Id1 inhibition by SFs was regulated by Akt, Src and a7nAChR (Figure 6).

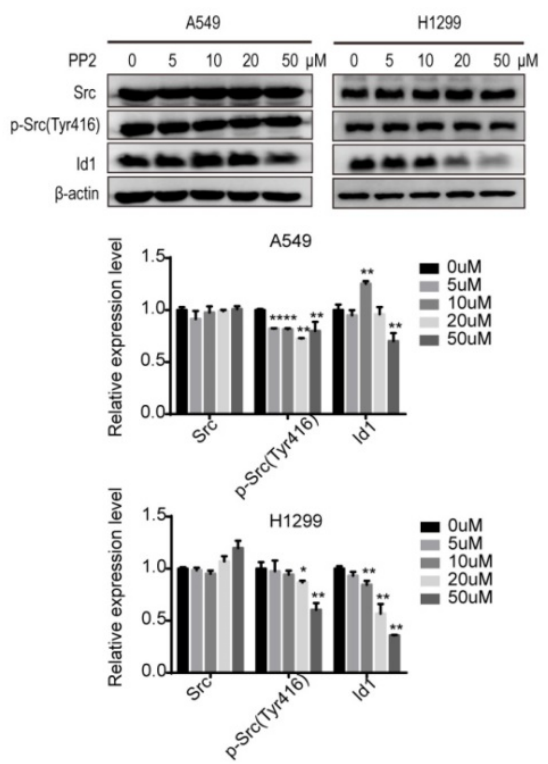



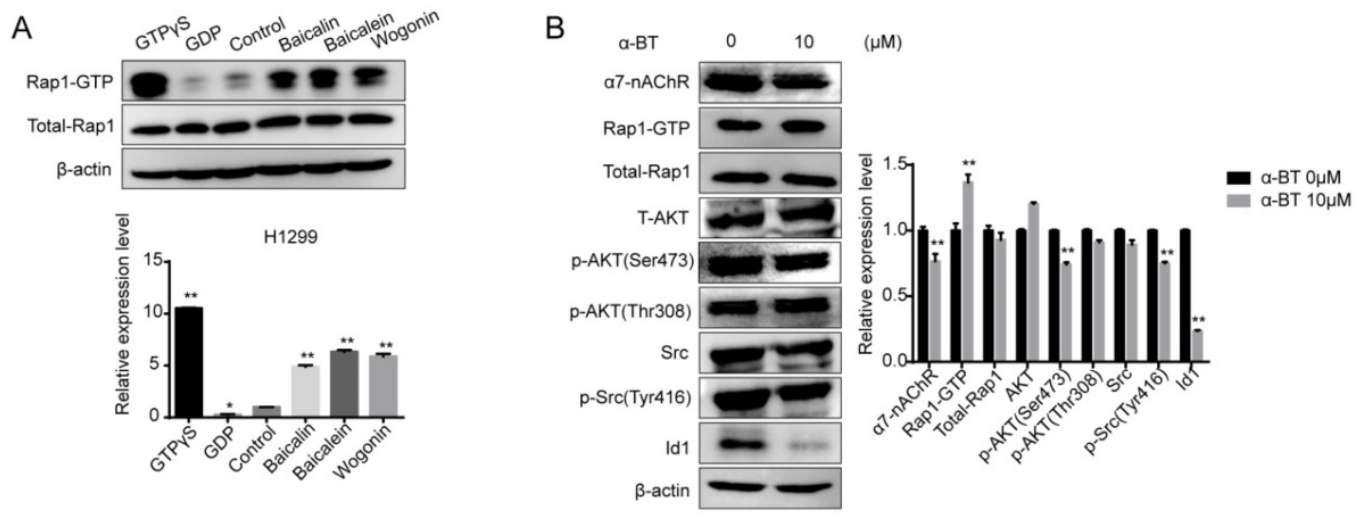

C
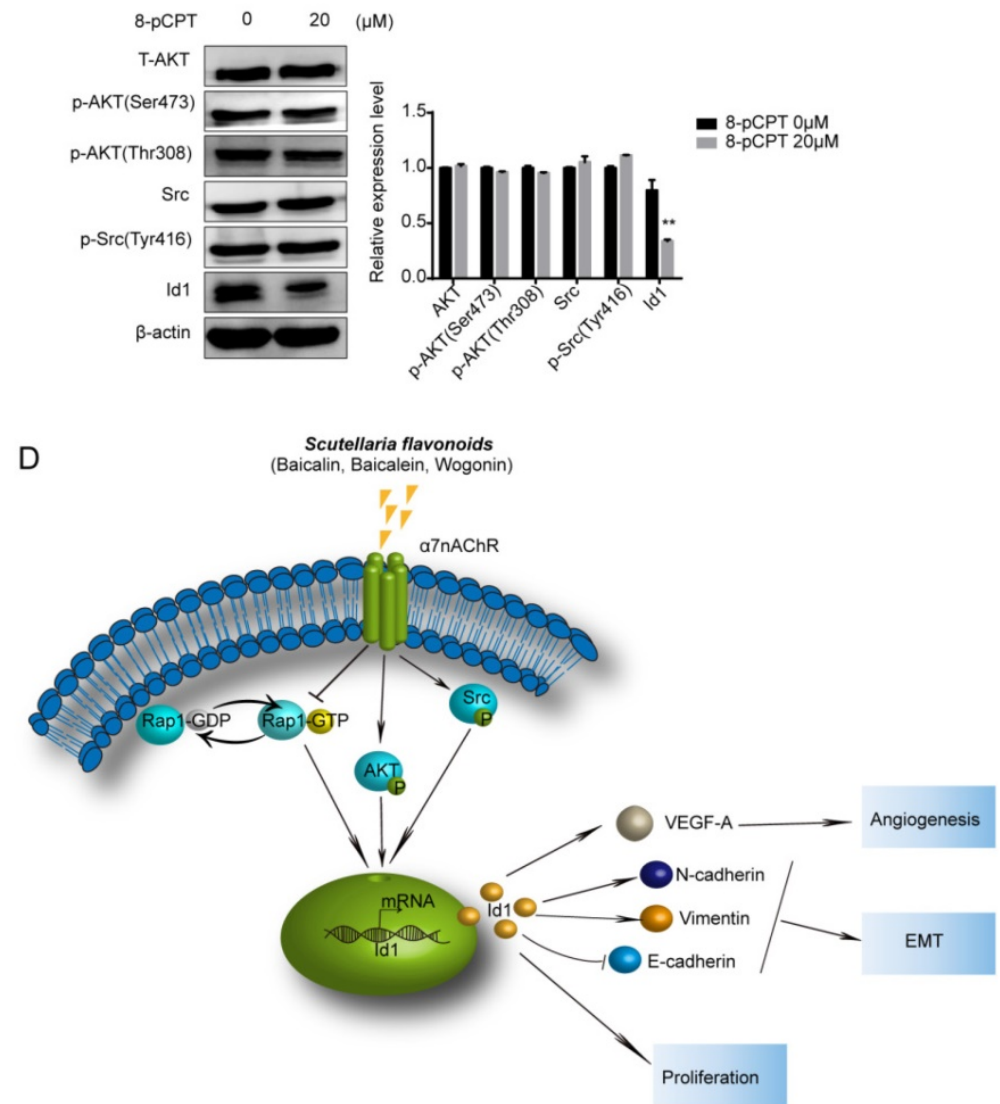

Figure 7. Rapl activation and Src/Akt dephosphorylation were suppressed by $\alpha 7 n A C h R$. (A) $H 1299$ cells were treated with or without baicalin, baicalein and wogonin for 24 h. Cell lysates of positive control or negative control were treated with GTPYS or GDP for 15 min respectively. Rap 1-GTP was analyzed using an immunoprecipitation kit. (B) $10 \mu \mathrm{M} \alpha-B T$ was added to $\mathrm{H} 1299$ cells and expressions of $\alpha 7 n A C h R$, Total-Rapl, T-Akt, p-Akt, Src, p-Src and Id1 were analyzed using western blot and Rapl-GTP was tested

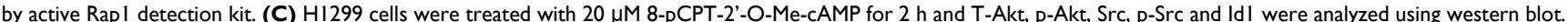
(D) A proposed mechanism by which SFs inhibit $\alpha 7 n A C h R$ to decrease Idl expression, thereby inhibiting proliferation, angiogenesis and EMT of NSCLC. Data represented mean \pm SEM. *, $p<0.05 ; * *, p<0.01$.

As KEGG pathway analysis demonstrated that Rap1 acts as an adaptor molecule of Src, Akt and Id1 (data not shown), we determined whether SFs -mediated down-regulation of Id1 was associated with Rap1. Rap1, a small GTPase that belongs to the Ras family of GTPases [23], cycles between a GTP-bound (active) and a GDP-bound (inactive) conformation, thereby allowing signaling pathways to be quickly switched on or off [41]. It is a regulator of morphogenesis which can establish cellular polarity, cell-matrix interactions and cell-cell adhesion [42]. Interestingly, the role of Rap1 in malignancy differs according to the cancer types [43]. As to glioma, Rap1 activity is required for the integrity of the glioma perivascular niche and glioma aggressiveness [44]. However, activation of Rap1 augments cisplatin-induced apoptosis by mediating inhibition of HDAC8 degradation in H1299 lung cancer cells [45]. In our study, we found baicalin, baicalein and wogonin significantly promoted the Rap1-GTP 
binding. As Rap1 is a Ras related protein and Ras gene is a well-known oncogene in NSCLC as well as an effector of nAChR [46], we presumed Rap1 was regulated by $\mathrm{a} 7 \mathrm{nAChR}$. Our data revealed that inhibition of a7nAChR with a-BT increased Rap1-GTP and dephosphorylated Src and Akt, with a concomitant decrease in Id1 expression. Down-regulation of Id1 by Rap1 activation in H1299 cells is consistent with previous observation in human dermal microvascular endothelial cells (HMVECs) [47]. However, different from KEGG pathway analysis, Src and Akt were not regulated by Rap1.This is the first report that Rap1 was found to be regulated by a7nAChR.

\section{Conclusion}

In conclusion, SFs inhibit the growth and metastasis of NSCLC and significantly reduce Id1 protein level in vivo and in vitro. Here, we introduce the activation of Rap1 and dephosphorylation of Akt and $\mathrm{Src}$ by a7nAChR repression as a novel mechanism by which SFs modulates Id1, consequently triggering the blockage of proliferation, EMT and angiogenesis (Figure 7D). Our results shed light on the mechanism of antitumor action of SFs which may benefit the development and promotion of traditional Chinese medicine and provide more inspirations for researchers in treating patients with NSCLC.

\section{Supplementary Material}

Supplementary figures.

http://www.ijbs.com/v15p1500s1.pdf

\section{Acknowledgments}

This study was funded by grants from National Natural Science Foundation of China (Grant No. 81403148, 81703829, 81673916, 81503518 and 81703829), Shanghai Municipal Commission of Health and Family Planning (ZYKC201602001), and Development Project of Shanghai Peak Disciplines Integrative Medicine (Grant No. 20150407).

\section{Authors' Contributions}

Conceptualization, W.G., J.D., Z.Z.; Methodology, Z.Z., B.L., L.L. (Linwei Lu), L.L. (Lumei Liu); Data acquisition, Z.Z., J.S., J.Q., Q.L., C.Y., S.J., N,M.;. Formal analysis, Z.Z., W.M., M.L; Writing, review and editing, Z.Z., W.G. Supervision, J.D., W.G.

\section{Competing Interests}

The authors have declared that no competing interest exists.

\section{References}

[1] Torre LA, Bray F, Siegel RL, et al. Global cancer statistics, 2012. CA Cancer J Clin. 2015; 65: 87-108.

[2] Johnson DH, Schiller JH. Novel therapies for the treatment of non-small cell lung cancer. Cancer chemotherapy and biological response modifiers. 2002; 20: 763-786.

[3] Tamanoi F, Hu TY. Non-small cell lung cancer, version 5.2017 clinical practice guidelines in oncology. J Natl Compr Canc Netw. 2017; 15: 504-535.

[4] Perk J, Iavarone A, Benezra R. Id family of helix-loop-helix proteins in cancer. Nat Rev Cancer. 2005; 5: 603-614.

[5] Rothschild SI, Kappeler A, Ratschiller D et al. The stem cell gene "inhibitor of differentiation 1" (ID1) is frequently expressed in non-small cell lung cancer. Lung Cancer. 2011; 71: 306-311;

[6] Roschger C, Cabrele C. The Id-protein family in developmental and cancer-associated pathways. Cell Commun Signal. 2017; 15: 7 .

[7] Pillai S, Rizwani W, Li X et al. ID1 facilitates the growth and metastasis of non-small cell lung cancer in response to nicotinic acetylcholine receptor and epidermal growth factor receptor signaling. Mol Cell Biol. 2011; 31: 3052-3067.

[8] Nair S, Bora-Singhal N, Perumal D et al. nicotine-mediated invasion and migration of non-small cell lung carcinoma cells by modulating STMN3 and GSPT1 genes in an ID1-dependent manner. Mol Cancer. 2014; 13: 173.

[9] Cheng YJ, Tsai JW, Hsieh KC et al. Id1 promotes lung cancer cell proliferation and tumor growth through Akt-related pathway. Cancer Lett. 2011; 307: 191-199.

[10] Rothschild SI, Tschan MP, Federzoni EA et al. MicroRNA-29b is involved in the Src-ID1 signaling pathway and is dysregulated in human lung adenocarcinoma. Oncogene. 2012; 31: 4221-4232.

[11] Lamer-Zarawska E, Wisniewska A, Blach-Olszewska Z. Anticancer properties of Scutellaria Baicalensis root in aspect of innate immunity regulation. Adv Clin Exp Med. 2010; 19: 419-428.

[12] Parekh HS, Liu G, Wei MQ. A new dawn for the use of traditional chinese medicine in cancer therapy. Mol Cancer. 2009; 8: 21

[13] Gong WY, Zhao ZX, Liu BJ et al. Exploring the chemopreventive properties and perspectives of baicalin and its aglycone baicalein in solid tumors. Eur $J$ Med Chem. 2017; 126: 844-852.

[14] Gong WY, Wu JF, Liu BJ et al. Flavonoid components in Scutellaria Baicalensis inhibit nicotine-induced proliferation, metastasis and lung cancer-associated inflammation in vitro. Int J Oncol. 2014; 44: 1561-1570.

[15] Mahmood MQ, Ward C, Muller HK et al. Epithelial mesenchymal transition (EMT) and non-small cell lung cancer (NSCLC): a mutual association with airway disease. Med Oncol. 2017; 34: 45 .

[16] Paleari L, Sessa F, Catassi A et al. Inhibition of non-neuronal alpha7-nicotinic receptor reduces tumorigenicity in A549 NSCLC xenografts. Int J Cancer. 2009; 125: 199-211.

[17] Giaccone G, Zucali PA. Src as a potential therapeutic target in non-small-cell lung cancer. Ann Oncol. 2008; 19: 1219-1223.

[18] Tsurutani J, Fukuoka J, Tsurutani H et al. Evaluation of two phosphorylation sites improves the prognostic significance of Akt activation in non-small-cell lung cancer tumors. J Clin Oncol. 2006; 24: 306-314.

[19] Kitayama H, Sugimoto $Y$, Matsuzaki $T$ et al. A ras-related gene with transformation suppressor activity. Cell. 1989; 56: 77-84.

[20] Bos JL. Linking rap to cell adhesion. Curr Opin Cell Biol. 2005; 17: 123-128.

[21] Retta SF, Balzac F, Avolio M. Rap1: a Turnabout for the crosstalk between cadherins and integrins. Eur J Cell Biol. 2006; 85: 283-293.

[22] Grando SA. Connections of nicotine to cancer. Nat Rev Cancer. 2014; 14: $419-429$.

[23] Shah S, Brock EJ, Ji K et al. Ras and Rap1: A tale of two GTPases. Semin Cancer Biol. 2019; 54: 29-39.

[24] Tsou LK., Lara-Tejero M, RoseFigura J et al. Antibacterial flavonoids from medicinal plants covalently inactivate type III protein secretion substrates. J Am Chem Soc. 2016; 138: 2209-2218.

[25] Shao ZH, Li CQ, Vanden HT et al. Extract from Scutellaria Baicalensis Georgi attenuates oxidant stress in cardiomyocytes. J Mol Cell Cardiol. 1999; 31: 1885-1895.

[26] Cheng CS, Chen J, Tan HY et al. Scutellaria Baicalensis and cancer treatment: recent progress and perspectives in biomedical and clinical studies. Am J Chin Med. 2018: 46: 25-54

[27] You J, Cheng J, Yu B et al. Baicalin, a Chinese herbal medicine, inhibits the proliferation and migration of human non-Small cell lung carcinoma (NSCLC) cells, A549 and H1299, by activating the SIRT1/AMPK signaling pathway. Med Sci Monit. 2018; 24: 2126-2133.

[28] Su G, Chen H, Sun X. Baicalein suppresses non-small cell lung cancer cell proliferation, invasion and Notch signaling pathway. Cancer Biomark. 2018; 22: $13-18$

[29] Lu C, Wang H, Chen $\mathrm{S}$ et al. Baicalein inhibits cell growth and increases cisplatin sensitivity of A549 and H460 cells via miR-424-3p and targeting PTEN/PI3K/Akt pathway. J Cell Mol Med. 2018; 22: 2478-2487.

[30] Chen G, Gharib TG, Huang CC et al. Discordant protein and mRNA expression in lung adenocarcinomas. Mol Cell Proteomics. 2002; 1: 304-313.

[31] Fong S, Debs RJ, Desprez P. Id genes and proteins as promising targets in cancer therapy. Trends Mol Med. 2004; 10: 387-392.

[32] Norton JD. Id Helix-loop-helix proteins in cell growth, differentiation and tumorigenesis. J Cell Sci. 2000; 113: 3897-3905. 
[33] Lyden D, Young AZ, Zagzag D et al. Id1 and Id3 are required for neurogenesis, angiogenesis and vascularization of tumour xenografts. Nature. 1999; 401: 670-677.

[34] Castanon E, Soltermann A, Lopez I et al. The inhibitor of differentiation-1 (Id1) enables lung cancer liver colonization through activation of an EMT program in tumor cells and establishment of the pre-metastatic niche. Cancer Lett. 2017; 402: 43-51.

[35] Young VJ, Ahmad SF, Brown JK et al. Peritoneal VEGF-A expression is regulated by TGF-beta1 through an ID1 pathway in women with endometriosis. Sci Rep. 2015; 5: 16859.

[36] Li DN, Wang L, Wang L et al. Expression of inhibitor of differentiation-1 and its effects on angiogenesis in gastric cancer. Cancer Biother Radiopharm. 2016; 31: 233-237.

[37] Wang G, Qiu J, Hu J et al. Id1: A novel therapeutic target for patients with atherosclerotic plaque rupture. Med Hypotheses. 2011; 76: 627-628.

[38] Grozio A, Paleari L, Catassi A et al. Natural agents targeting the alpha7-nicotinic-receptor in NSCLC: a promising prospective in anti-cancer drug development. Int J Cancer. 2008; 122: 1911-1915.

[39] Dasgupta P, Rastogi S, Pillai S et al. Nicotine induces cell proliferation by beta-arrestin-mediated activation of Src and Rb-Raf-1 pathways. J Clin Invest. 2006; 116: 2208-2217.

[40] Gautschi O, Tepper CG, Purnell PR et al. Regulation of Id1 expression by SRC: implications for targeting of the bone morphogenetic protein pathway in cancer. Cancer Res. 2008; 68: 2250-2258.

[41] Pannekoek W, Kooistra MRH, Zwartkruis FJT et al. Cell-cell junction formation: the role of rap1 and rap1 guanine nucleotide exchange factors. Biochimica Biophysica Acta (BBA) - Biomembranes. 2009; 1788: 790-796.

[42] Frische EW, Zwartkruis FJT. Rap1, a mercenary among the ras-like GTPases. Dev Biol. 2010; 340: 1-9.

[43] Hattori M. Rap1 GTPase: functions, regulation, and malignancy. J Biochem. 2003; 134: 479-484.

[44] Niola F, Zhao X, Singh D et al. Mesenchymal high-grade glioma is maintained by the ID-RAP1 axis. J Clin Invest. 2013; 123: 405-417.

[45] Park J, Juhnn Y. cAMP signaling increases histone deacetylase 8 expression via the epac2-rap1-Akt pathway in H1299 lung cancer cells. Experimental \& Molecular Medicine. 2017; 49: e297.

[46] Arredondo J, Chernyavsky AI, Jolkovsky DL et al. Receptor-mediated tobacco toxicity: cooperation of the Ras/Raf-1/MEK1/ERK and JAK-2/STAT-3 pathways downstream of alpha7 nicotinic receptor in oral keratinocytes. Faseb J. 2006; 20: 2093-2101.

[47] Doebele RC, Schulze-Hoepfner FT, Hong J et al. A novel interplay between Epac/Rap1 and mitogen-activated protein kinase 5/extracellular signal-regulated kinase 5 (MEK5/ERK5) regulates thrombospondin to control angiogenesis. Blood. 2009; 114: 4592-4600. 